\title{
Transthyretin regulates the migration and invasion of JEG-3 cells
}

\author{
LIYUN GONG, LEI ZHU, SHUZHEN WANG and ZHENYU ZHANG
}

Department of Obstetrics and Gynecology, Chao-Yang Hospital, Capital Medical University, Beijing 100020, P.R. China

Received June 30, 2016; Accepted November 28, 2016

DOI: $10.3892 / 01.2016 .5545$

\begin{abstract}
Preeclampsia (PE) is a pregnancy-specific disorder characterized by new-onset hypertension and proteinuria that occurs after 20 weeks of gestation. It involves several organs and continues to be a leading cause of maternal and perinatal morbidity and mortality worldwide. Shallow trophoblast invasion is a common pathological feature of PE. Transthyretin (TTR) is a $56-\mathrm{kDa}$ homotetrameric protein that binds thyroid hormone and retinol binding protein. Dysregulated TTR expression has been found in cases of PE. The aim of the present study was to determine the functional role of TTR in the migration and invasion of JEG-3 choriocarcinoma cells. JEG-3 cells were transfected with a plasmid construct expressing TTR (pCMV-Myc-TTR) or an empty plasmid (pCMV-Myc). Cell migration and invasion capacities were assessed by Transwell migration and invasion assays, respectively. These experiments demonstrated that TTR overexpression significantly increased the migration and invasion potential of JEG-3 cells. Matrix metalloproteinases (MMPs) are a family of zinc-containing endopeptidases capable of degrading a wide range of extracellular matrix components. Western blot analysis revealed that TTR overexpression resulted in significantly increased levels of MMP2 and MMP9 in JEG-3 cells. In conclusion, our findings suggest an important role for TTR in regulating trophoblast invasion and migration, representing a possible underlying pathological and molecular mechanisms of PE.
\end{abstract}

\section{Introduction}

Preeclampsia (PE) is a pregnancy-specific disorder characterized by new-onset hypertension and proteinuria that occurs after 20 weeks of gestation. It is a systemic disease that involves several organs such as the brain, liver, and

Correspondence to: Professor Zhenyu Zhang, Department of Obstetrics and Gynecology, Chao-Yang Hospital, Capital Medical University, 8 Gongti South Road, Chaoyang, Beijing 100020, P.R. China

E-mail: zhenyuzhang2000@163.com

Key words: transthyretin, trophoblast invasion, preeclampsia, matrix metalloproteinase kidney, as well as the coagulation system (1). This disorder affects $2-8 \%$ of all pregnancies and continues to be a leading cause of maternal and perinatal morbidity and mortality worldwide $(2,3)$. Although the specific etiology and pathogenesis of PE remain unknown, it is widely accepted that PE is associated with inadequate invasion of trophocytes, and spiral artery remodeling (4). In normal pregnancies, extravillous cytotrophoblasts of fetal origin invade the uterine spiral arteries of the decidua and myometrium. These invasive cytotrophoblasts replace the endothelial layer of the maternal spiral arteries, transforming them from small, high-resistance vessels into large-caliber vessels. However, in the development of preeclamptic placenta, reduced invasive ability results in trophocytes failing to invade the deep layers of the myometrium and thus failing to appropriately remodel the uterine spiral arteries (5). Consequently, the transformation of spiral arteries from high-resistance, low-flow vessels into large-caliber vessels is suppressed. The decreased blood flow and fetoplacental perfusion leads to placental hypoxia and ischemia, subsequent systemic endothelial dysfunction and PE (6). However, the molecular mechanisms for the regulation of trophoblast behavior remain largely elusive.

Transthyretin (TTR), formerly known as pre-albumin, was first discovered in 1942, both in human cerebrospinal fluid and blood. Subsequently, TTR synthesis has been identified in the yolk sac, placenta, pancreas and intestine of humans. It is a 56-kDa homotetrameric protein that binds thyroid hormone and retinol binding protein. Dysregulated placental TTR has been found in cases of intrauterine growth restriction and severe early onset PE (7). While a causative role of TTR in PE has been postulated (8), the precise pathogenic mechanisms of $\mathrm{PE}$ have not yet been clarified. Our study aimed to investigate the possible role of TTR in the pathophysiology of PE and its function in trophoblast biology.

\section{Materials and methods}

Cell culture. The human choriocarcinoma cell line, JEG-3, was obtained from the Cancer Hospital, Chinese Academy of Medical Sciences (Beijing, China). JEG-3 cells were cultured in RPMI-1640 medium supplemented with $10 \%$ fetal bovine serum (FBS; Gibco-BRL, Carlsbad, CA, USA) at $37^{\circ} \mathrm{C}$ in a $5 \% \mathrm{CO}_{2}$ incubator. When the cells reached $80-90 \%$ confluence, they were trypsinized and subcultured into new culture flasks.

Construction of a recombinant plasmid overexpressing TTR. The nucleotide sequence of the coding region of the TTR gene 
was used to design amplification primers with Primer Premier 5 software (Premier Biosoft, Palo Alto, CA, USA). The primer sequences were: forward, 5'-GTAGAATTCGGATGGCTTCT CATCGTCTG-3' and reverse, 5'-GTAGGTACCTCATTCCTT GGGATTGGTG-3'. Human cDNA was used as a template to amplify the coding region of the TTR gene. The resulting PCR product was ligated with the EcoRI/KpnI enzyme restriction sites of the pCMV-Myc plasmid. The positive recombinant plasmid was identified by double enzyme digestion and the sequence was termed pCMV-Myc-TTR. The empty plasmid (pCMV-Myc) was used as a negative control. JEG-3 cells were seeded in 6-well plates and allowed to grow to $80-90 \%$ confluence. Transfection was performed using the Lipofectamine 2000 reagent (Invitrogen Life Technologies, Carlsbad, CA, USA) according to the manufacturer's instructions. The medium was replaced with fresh culture medium $6 \mathrm{~h}$ after transfection.

Western blot analysis. Protein extracts were prepared from cells at $48 \mathrm{~h}$ post-transfection using ice-cold radioimmunoprecipitation assay lysis buffer (Beyotime Institute of Biotechnology, Haimen, China) and protease inhibitor cocktail (Sigma-Aldrich, St. Louis, MO, USA). Protein concentrations were determined using a bicinchoninic acid protein assay kit (Beyotime Institute of Biotechnology). Equal amounts of protein were mixed with loading buffer and boiled at $100^{\circ} \mathrm{C}$ for $3-5 \mathrm{~min}$, chilled on ice, and resolved by $12 \%$ sodium dodecyl sulfate-polyacrylamide gel electrophoresis (SDS-PAGE). The separated proteins were then electrophoretically transferred to $0.45-\mu \mathrm{m}$ polyvinylidene fluoride (PVDF) membranes (Millipore, Bedford, MA, USA) using a semi-dry western blot transfer system (Bio-Rad Laboratories, Inc., Hercules, CA, USA) for $90 \mathrm{~min}$ at $200 \mathrm{~mA}$. The membranes were subsequently washed with TBST (TBS containing 0.1\% Tween-20) for $1 \mathrm{~min}$, after blocking with $5 \%$ non-fat dry milk for $1 \mathrm{~h}$ at room temperature. The membranes were then sequentially incubated overnight at $4{ }^{\circ} \mathrm{C}$ with rabbit anti-prealbumin monoclonal antibody (ab199074, 1:2,000 diluted), rabbit anti-matrix metalloproteinase 2 (MMP2) monoclonal antibody (ab92536, 1:1,000 diluted) and rabbit anti-MMP9 monoclonal antibody (ab76003, 1:4,000 diluted) (all from Abcam, Cambridge, MA, USA), and anti-GAPDH (no. 2118, 1:5,000 diluted; Cell Signaling Technology, Inc., Danvers, MA, USA) which was used as the internal control. The following day, the membranes were washed three times in TBST (5 min each) and incubated with a secondary HRP-conjugated goat anti-rabbit antibody (no. 7074, 1:10,000 diluted; Cell Signaling Technology, Inc.) for $1 \mathrm{~h}$ at room temperature. Finally, immunoreactive bands were detected with an enhanced chemiluminescence kit (Millipore) and exposed to X-ray film.

Transwell migration assay. The Transwell migration assay was performed by determining the ability of cells to invade the $8-\mu \mathrm{m}$ pores of polycarbonate membranes (cat. no. 3422; Corning Costar Inc., Corning, NY, USA), which were placed at the bottom of Transwell chambers in 24-well plates. JEG-3 cells were transfected with pCMV-Myc-TTR or the empty plasmid when $80-90 \%$ confluent. After 24 h of incubation, $1 \times 10^{5}$ JEG-3 cells were resuspended in $100 \mu$ l serum-free medium containing $1 \%$ bovine serum albumin and were plated on the top of the chamber and incubated with $650 \mu \mathrm{l}$

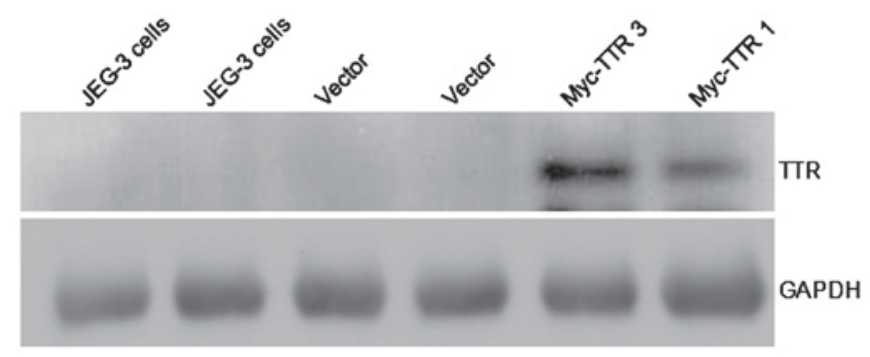

Figure 1. Overexpression of TTR by western blot analysis. Western blot analysis revealed differences in TTR protein expression in JEG-3 cells transfected with the TTR plasmid construct or empty plasmid and untransfected JEG-3 cells. There was no expression of TTR in the JEG-3 cells transfected with the empty plasmid and untransfected JEG-3 cells, TTR protein was overexpressed after transfection with pCMV-Myc-TTR. TTR expression was increased in cells transfected with $3 \mu \mathrm{g}$ Myc-TTR construct, compared with $1 \mu \mathrm{g}$. TTR 1 , the quantity of TTR construct plasmid is $1 \mu \mathrm{g}$; TTR 3 , the quantity of TTR construct plasmid is $3 \mu \mathrm{g}$. TTR, transthyretin.

culture medium containing $10 \%$ FBS in the bottom chamber. The cells with or without TTR overexpression were divided into two groups, and treated in the presence or absence of $1 \mu \mathrm{mol} / 1$ levothyroxine $\left(\mathrm{L}-\mathrm{T}_{4}\right)$. After incubation at $37^{\circ} \mathrm{C}$ in an atmosphere of $5 \% \mathrm{CO}_{2}$ for $24 \mathrm{~h}$, the cells on the top surface of the Transwell membrane were wiped off with cotton swabs. Subsequently, the cells on the bottom surface of the membrane were fixed with $95 \%$ ethyl alcohol for $30 \mathrm{~min}$ and stained with $0.1 \%$ crystal violet for 3-5 min at room temperature (Beijing Solarbio Science \& Technology Co., Ltd., Beijing, China). The invaded cells on the underside of the membrane were enumerated using an inverted microscope in five random fields at x 50 magnification (IX-71; Olympus, Tokyo, Japan). The results are presented as mean of invaded cells \pm standard deviation (SD) and each assay was repeated at least 3 times.

Matrigel-based Transwell invasion assay. A Matrigel-based Transwell invasion assay was performed to assess the invasion ability of JEG-3 cells, $24 \mathrm{~h}$ after transfection. The method was similar to the cell migration assay, except that the membrane was pre-treated with $60 \mu 1$ Matrigel (BD Biosciences, Franklin Lakes, NJ, USA) at a concentration of $2 \mathrm{mg} / \mathrm{ml}$, and the results were observed after incubation for $36 \mathrm{~h}$. Experiments were performed in triplicate and the results are presented as mean of invaded cells \pm SD.

Statistical analysis. Data are presented as mean \pm SD. One-way analysis of variance (ANOVA) was conducted to compare multiple groups, followed by the Bonferroni post hoc test for the comparisons between groups. GraphPad Prism software, version 4.0 (GraphPad Software, Inc., San Diego, CA, USA), was used for data analysis and plotting. $\mathrm{P}<0.05$ was considered to indicate a statistically significant difference.

\section{Results}

TTR protein expression after recombinant plasmid transfection. JEG-3 cells were transfected with pCMV-Myc-TTR or empty plasmid. Myc-TTR was overexpressed in JEG-3 cells transfected with pCMV-Myc-TTR as determined by western blot analysis, whereas there was no expression of TTR protein 


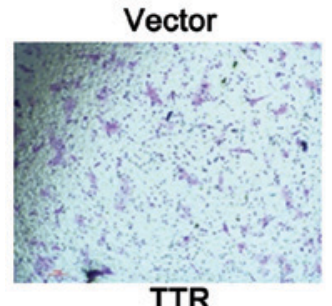

TTR

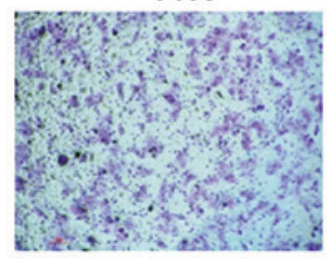

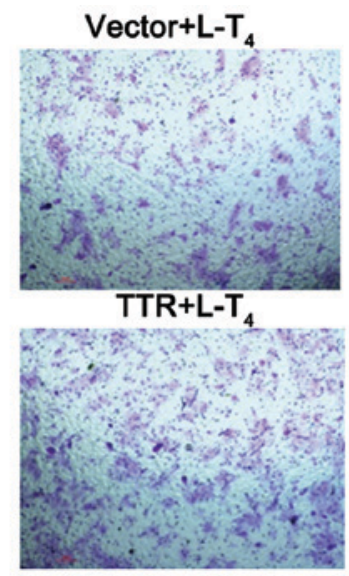

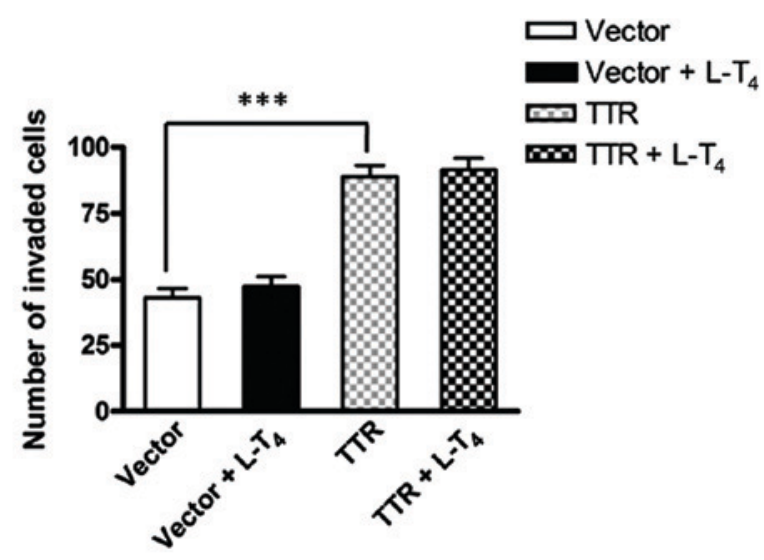

Figure 2. TTR overexpression promotes the migration ability of JEG-3 cells in the 24-h migration assay. Transwell migration assay was performed to assess the migration ability of JEG-3 cells with and without TTR overexpression. Cells with or without TTR overexpression were divided into two groups (in the presence or absence of $1 \mu \mathrm{mol} / 1 \mathrm{~L}-\mathrm{T}_{4}$ ). The invaded cells on the underside of the membrane were enumerated using an inverted microscope in 5 random fields. At x 50 magnification; scale bar, $100 \mu \mathrm{m}$. Experiments were performed in triplicate and the results are presented as mean of invaded cells $\pm \mathrm{SD},{ }^{* * * *} \mathrm{P}<0.001$ between the two groups. TTR, transthyretin; $\mathrm{L}_{-} \mathrm{T}_{4}$, levothyroxine.

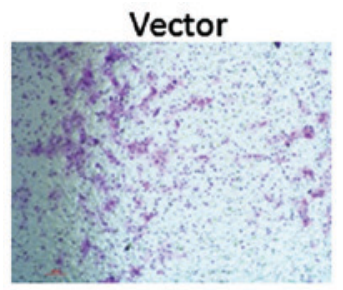

TTR

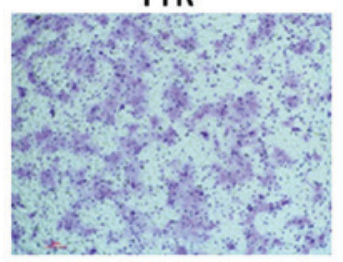

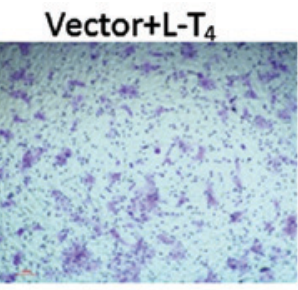

TTR+L-T

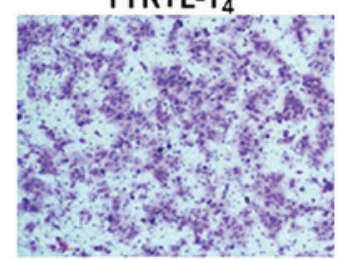

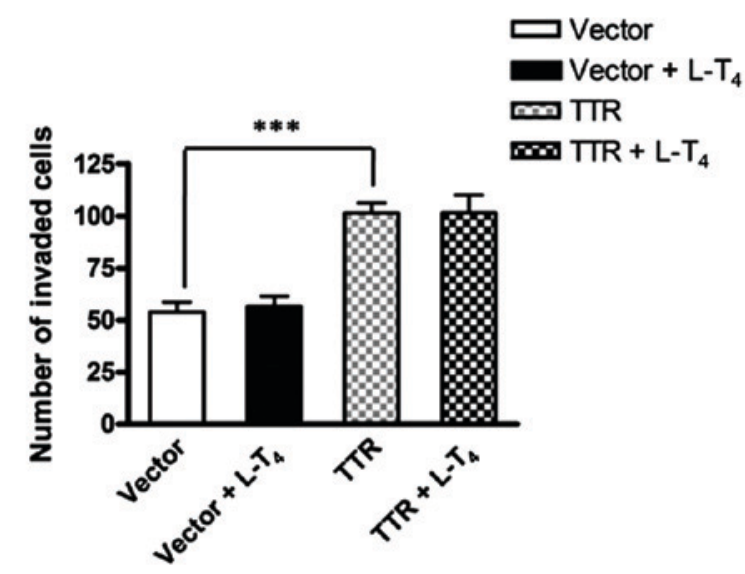

Figure 3. TTR overexpression promotes the invasion of JEG-3 cells in the 36-h invasion assay. Matrigel-based Transwell invasion assay was performed to assess the invasion ability of JEG-3 with and without TTR overexpression. Cells with or without TTR overexpression were divided into two groups (in the presence or absence of $1 \mu \mathrm{mol} / 1 \mathrm{~L}-\mathrm{T}_{4}$ ). The invaded cells on the underside of the membrane were enumerated using an inverted microscope in five random fields. At $\mathrm{x} 50$ magnification; scale bar, $100 \mu \mathrm{m}$. Experiments were performed in triplicate and the results are presented as mean of invaded cells $\pm \mathrm{SD},{ }^{* * *} \mathrm{P}<0.001$ between the two groups. TTR, transthyretin; $\mathrm{L}-\mathrm{T}_{4}$, levothyroxine.

in JEG-3 cells transfected with the empty plasmid. These data indicated that JEG-3 cells expressed extremely low levels of endogenous TTR protein (Fig. 1).

Overexpression of TTR promotes the migration and invasion of JEG-3 cells. The migration and invasion ability of JEG-3 cells transfected with the TTR-expressing construct or empty plasmid were assessed by a Transwell migration assay and Matrigel-based invasion assay, respectively. In the 24-h migration assay, the number of invaded cells increased significantly when JEG-3 cells were transfected with pCMV-Myc-TTR compared with the empty plasmid. However, in TTR-overexpressing cells, there was no significant difference in cell invasion in the presence or absence of

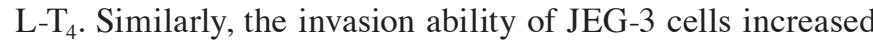
significantly after TTR overexpression, compared with the control cells in the 36-h invasion assay. However, there was no difference in cell invasion between TTR-overexpressing cells in the presence or absence of L-T $\mathrm{T}_{4}$ (Figs. 2 and 3).
Overexpression of TTR upregulates MMP2 and MMP9. MMPs are a family of proteolytic enzymes that have been implicated in extracellular matrix remodeling in the process of trophocyte invasion. To investigate the molecular mechanisms of TTR in mediating trophoblast invasion, we used western blot analysis to analyze the protein levels of MMP2 and MMP9 at $48 \mathrm{~h}$ after transfection. The results showed that overexpression of TTR significantly increased the expression levels of MMP2 and MMP9 (Fig. 4), indicating that TTR likely regulates the invasion ability of these cells through MMP-mediated remodeling of extracellular matrix.

\section{Discussion}

JEG-3 is a line of choriocarcinoma cells that originated from choriocarcinoma explants. They are phenotypically similar to extravillous trophoblasts (EVTs). JEG-3 cells express human choriogonadotropin; human leukocyte antigen-G, integrin $\alpha$ (ITGA)1, 5 and 6; and MMP2 and MMP9; and have 

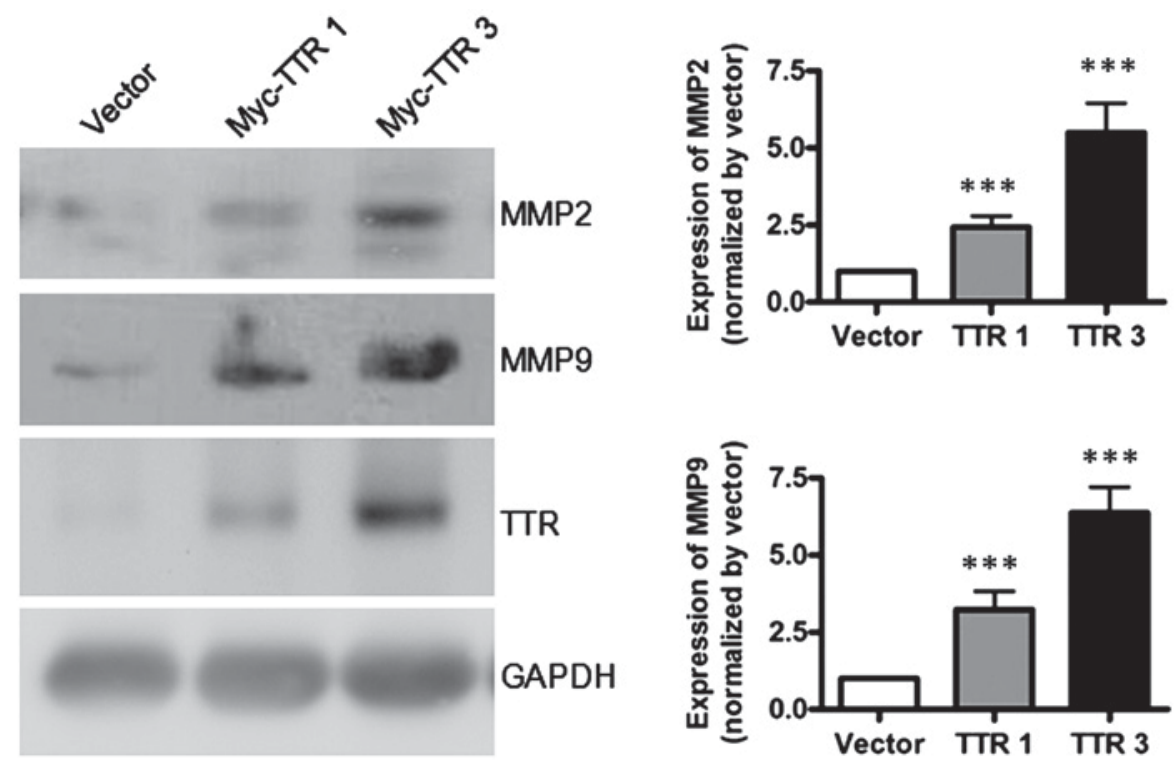

Figure 4. Protein levels of MMP2 and MMP9 in JEG-3 cells transfected with Myc-TTR and empty plasmid were assessed by western blot analysis. The blots shown are representative of three independent experiments. The overexpression of TTR significantly increased the protein level of MMP2 and MMP9. The levels of MMP2 and MMP9 were higher in cells transfected with $3 \mu \mathrm{g}$ Myc-TTR construct compared with $1 \mu \mathrm{g}$. Columns show mean $\pm \mathrm{SD}$, ${ }^{* * *} \mathrm{P}<0.001$ between groups. TTR 1 , the quantity of TTR construct plasmid is $1 \mu \mathrm{g}$; TTR 3 , the quantity of TTR construct plasmid is $3 \mu \mathrm{g}$. MMPs, matrix metalloproteinases.

been extensively used as an in vitro model to study the properties of trophoblast migration and invasion $(9,10)$.

The process of EVT cell invasion of the maternal spiral arteries is critical for the establishment of a successful pregnancy and is very similar to the invasion of tumor cells $(11,12)$. Failure of the process has been recognized as a common pathologic feature of PE $(13,14)$. In addition, this process is regulated by many cytokines and chemokines (15-17). In the placentas of patients with $\mathrm{PE}$, trophoblast dysfunction reduces the invasion and migration capacities of EVTs. When the invasion of trophocytes into the endometrium at the maternal-fetal interface of PE patients is very shallow, and the vascular endothelium has not been replaced by trophocytes, this will result in placental hypoperfusion, hypoxia, or ischemia and thus induce the occurrence and deterioration of PE (18).

It is widely believed that the pathophysiological changes of PE may result from the abnormal expression of certain proteins. A previous study of PE using surface-enhanced laser desorption ionization time-of-flight mass spectrometry (SELDI-TOF-MS) detected 10 significantly different protein peaks between patients with hypertensive disorders of pregnancy and healthy controls (18). Liu et al (19) used peptide ligand library affinity chromatography combined with 1D gel-LC-MS/MS analysis to identify proteins that were differentially expressed in sera of preeclamptic patients compared with sera from healthy pregnant women, and found that TTR was significantly downregulated in preeclamptic patients. Kalkunte et al (20) used SELDI-TOF-MS combined with two-dimensional gel electrophoresis to test sera collected from patients with PE or subjects with normal pregnancy and found reduced levels of TTR in PE serum. This study also demonstrated that TTR aggregates to form deposits in preeclamptic placental tissue and causes apoptosis. Furthermore, native TTR inhibited all PE-like features in the humanized mouse model, including new-onset proteinuria, increased blood pressure, glomerular endotheliosis and production of anti-angiogenic factors. Chen et al (8) assumed that TTR may cause a disorder of maternal vascular function and contribute to the pathology of PE by deposition of TTR amyloid fibrils in the vascular system, which are produced by variant TTR proteins, resulting in organ ischemia. The study by Zhu et al (21) using ELISA and western blot analysis revealed that TTR levels were markedly decreased in early onset severe PE cases compared with controls, and TTR levels were lower in the early onset PE patients than in the late onset group, indicating that changes in TTR levels may correlate with the severity of PE.

Previous studies suggested that decreased TTR expression may be responsible for the occurrence of PE (20,21). TTR levels may change prior to the onset of $\mathrm{PE}$ and may represent a candidate biomarker to predict PE. However, it remains unclear how TTR is involved in the pathogenesis of PE. The present study showed that the number of invaded cells increased significantly when JEG-3 cells were transfected with Myc-TTR compared with the empty plasmid, in both the Transwell migration assay and Matrigel-based invasion assay. This suggested that TTR can promote the migration and invasion ability of trophoblasts. Additionally, a decreased TTR expression leads to inhibition of the migration and invasion of trophoblasts in PE patients. Shallow trophoblast invasion in turn, results in placental hypoxia and ischemia, which eventually leads to the occurrence of PE. However, the present results also showed that there was no significant difference in the number of invaded TTR-overexpressing cells in the presence or absence of $\mathrm{L}_{-} \mathrm{T}_{4}$. It is known that TTR is a carrier protein. Its main function is the transport of $\mathrm{T}_{4}$ (19). However, we showed that the migration and invasion ability of JEG-3 cells was not


not exert its roles through the $\mathrm{TTR}-\mathrm{T}_{4}$ complex to promote the migration and invasion of trophoblasts. Our study further confirmed the role of TTR in the pathogenesis of PE, although further study is required to clarify the molecular mechanisms. 
MMPs are a family of zinc-containing endopeptidases capable of degrading a wide range of extracellular matrix components (22). Of these, MMP2 and MMP9, also known as gelatinase $\mathrm{A}$ and $\mathrm{B}$, respectively, are the most frequently investigated MMPs and are thought to play important roles in trophoblast invasion. It has been demonstrated that patients with PE exhibit low levels of placental MMP2 and MMP9 $(23,24)$. It has also been shown that MMPs are involved in the remodeling of uterine spiral arteries, and reduced MMP activity may affect trophoblastic invasion, which subsequently induces abnormal placental development and onset of PE (25). The present findings showed that overexpression of TTR significantly increased the protein levels of MMP2 and MMP9, indicating that TTR likely regulates the invasion ability of cells through MMP-mediated remodeling of extracellular matrix. More specifically, TTR likely promotes the migration and invasion ability of trophoblasts by increasing the secretion of MMP2 and MMP9. The reduced levels of TTR may decrease the secretion of MMP2 and MMP9 in PE patients and subsequently inhibit the migration and invasion ability of trophoblasts. Shallow trophoblast invasion contributes to placental hypoxia and ischemia, which eventually leads to the occurrence of PE.

In conclusion, we have demonstrated that overexpression of TTR effectively promotes the migration and invasion ability of JEG-3 cells, which was associated with increased protein levels of MMPs. Our findings support an important role for TTR in regulating trophoblast invasion and migration, representing a possible underlying pathological and molecular mechanism of PE.

\section{Acknowledgements}

The present study was supported by the Sino-RUS Cooperation Funds (no. 2015DFR31070) and by the National Natural Science Funds (no.81571455).

\section{References}

1. ACOG Committee on Obstetric Practice; American College of Obstetricians and Gynecologists: ACOG practice bulletin. Diagnosis and management of preeclampsia and eclampsia. Number 33, January 2002. Int J Gynaecol Obstet 77: 67-75, 2002.

2. Duley L, Meher S and Abalos E: Management of pre-eclampsia. BMJ 332: 463-468, 2006.

3. Poon LC and Nicolaides KH: Early prediction of preeclampsia. Obstet Gynecol Int 2014: 297397, 2014.

4. Tal R: The role of hypoxia and hypoxia-inducible factor-1alpha in preeclampsia pathogenesis. Biol Reprod 87: 134, 2012.

5. Goldman-Wohl D and Yagel S: Regulation of trophoblast invasion: from normal implantation to pre-eclampsia. Mol Cell Endocrinol 187: 233-238, 2002.

6. Lam C, Lim KH and Karumanchi SA: Circulating angiogenic factors in the pathogenesis and prediction of preeclampsia. Hypertension 46: 1077-1085, 2005.
7. Fruscalzo A, Schmitz R,Klockenbusch W,Köhler G,Londero AP, Siwetz M and Huppertz B: Human placental transthyretin in fetal growth restriction in combination with preeclampsia and the HELLP syndrome. Histochem Cell Biol 138: 925-932, 2012.

8. Chen Y and Zhang Z: Does transthyretin function as one of contributors for preeclampsia? Med Hypotheses 76: 8-10, 2011.

9. Huppertz B, Kertschanska S, Demir AY, Frank HG and Kaufmann P: Immunohistochemistry of matrix metalloproteinases (MMP), their substrates, and their inhibitors (TIMP) during trophoblast invasion in the human placenta. Cell Tissue Res 291: 133-148, 1998.

10. Hannan NJ, Paiva P, Dimitriadis E and Salamonsen LA: Models for study of human embryo implantation: choice of cell lines? Biol Reprod 82: 235-245, 2010.

11. Harris LK: Review: trophoblast-vascular cell interactions in early pregnancy: how to remodel a vessel. Placenta 31 (Suppl): S93-S98, 2010

12. Hiden U, Bilban M, Knöfler M and Desoye G: Kisspeptins and the placenta: regulation of trophoblast invasion. Rev Endocr Metab Disord 8: 31-39, 2007.

13. Pennington KA, Schlitt JM, Jackson DL, Schulz LC and Schust DJ: Preeclampsia: multiple approaches for a multifactorial disease. Dis Model Mech 5: 9-18, 2012.

14. Kaitu'u-Lino TJ, Ye L, Tuohey L, Dimitriadis E, Bulmer J, Rogers P, Menkhorst E, Van Sinderen M, Girling JE, Hannan N, et al: Corin, an enzyme with a putative role in spiral artery remodeling, is up-regulated in late secretory endometrium and first trimester decidua. Hum Reprod 28: 1172-1180, 2013.

15. Ren L, Liu YQ, Zhou WH and Zhang YZ: Trophoblast-derived chemokine CXCL12 promotes CXCR4 expression and invasion of human first-trimester decidual stromal cells. Hum Reprod 27: 366-374, 2012

16. Kim YJ: Pathogenesis and promising non-invasive markers for preeclampsia. Obstet Gynecol Sci 56: 2-7, 2013.

17. Liu X, Dai LI and Zhou R: Association between preeclampsia and the CXC chemokine family (Review). Exp Ther Med 9: 1572-1576, 2015

18. Gong LY, Zhang ZY, Zheng YH and Zhang JZ: Study of a serum protein fingerprint diagnostic model in patients with hypertensive disorder complicating pregnancy. Zhonghua Fu Chan Ke Za Zhi 42: 822-825, 2007 (In Chinese).

19. Liu C, Zhang N, Yu H, Chen Y, Liang Y, Deng H and Zhang Z: Proteomic analysis of human serum for finding pathogenic factors and potential biomarkers in preeclampsia. Placenta 32: 168-174, 2011.

20. Kalkunte SS, Neubeck S, Norris WE, Cheng SB, Kostadinov S Vu Hoang D, Ahmed A, von Eggeling F, Shaikh Z, Padbury J, et al: Transthyretin is dysregulated in preeclampsia, and its native form prevents the onset of disease in a preclinical mouse model. Am J Pathol 183: 1425-1436, 2013.

21. Zhu L, Chen Y, Liu C, Deng H, Zhang N, Wang S and Zhang Z: Transthyretin as a novel candidate biomarker for preeclampsia. Exp Ther Med 7: 1332-1336, 2014.

22. Heissig B, Hattori K, Friedrich M, Rafii S and Werb Z: Angiogenesis: vascular remodeling of the extracellular matrix involves metalloproteinases. Curr Opin Hematol 10: 136-141, 2003.

23. Omran OM, Shokry M, Ismail H, Omar G and Rezk M: Expression of matrix metalloproteinases 2 and 9 in human trophoblasts of normal and preeclamptic placentas. Int J Health Sci (Qassim) 5 (Suppl 1): 21-23, 2011.

24. Yang Y, Zhang J, Gong Y, Liu X, Bai Y, Xu W and Zhou R: Increased expression of prostasin contributes to early-onset severe preeclampsia through inhibiting trophoblast invasion. J Perinatol 35: 16-22, 2015.

25. Cockle JV, Gopichandran N, Walker JJ, Levene MI and Orsi NM: Matrix metalloproteinases and their tissue inhibitors in preterm perinatal complications. Reprod Sci 14: 629-645, 2007. 